\title{
A Kernel Representation of Dirac Structures for Infinite-dimensional Systems
}

\author{
O.V. Iftime ${ }^{1}$, M. Roman ${ }^{2}$, A. Sandovici ${ }^{2}{ }^{*}$ \\ ${ }^{1}$ Department of Economics, Econometrics and Finance, University of Groningen \\ Nettelbosje 2, 9747 AE, Groningen, The Netherlands \\ 2 Department of Mathematics, "Gheorghe Asachi" Technical University \\ B-dul Carol I, nr. 11, 700506, Iaşi, Romania
}

\begin{abstract}
Dirac structures are used as the underlying structure to mathematically formalize port-Hamiltonian systems. This note approaches the Dirac structures for infinite-dimensional systems using the theory of linear relations on Hilbert spaces. First, a kernel representation for a Dirac structure is proposed. The one-to-one correspondence between Dirac structures and unitary operators is revisited. Further, the proposed kernel representation and a scattering representation are constructively related. Several illustrative examples are also presented in the paper.
\end{abstract}

Keywords and phrases: Dirac structure, linear relation, Hilbert space, infinite-dimensional system

Mathematics Subject Classification: 93A30, 93B28

\section{Introduction}

Port-based modeling leads to port Hamiltonian systems which are defined with respect to a geometric structure, called Dirac structure (see for example [4] and the references therein). Dirac structures for finite dimensional systems were introduced by Courant [5] as a generalization of Poisson and pre-symplectic structures. Dorfman [7] presented for the first time the notion of infinite-dimensional Dirac structures in the context of nonlinear evolution equations. Port Hamiltonian infinite-dimensional systems have been proposed for distributed parameter systems with energy flow at their boundary by Maschke and van der Schaft [22]. Many efforts have been made during the last years towards understanding port Hamiltonian systems and the underlying Dirac structures in the context of modeling and control of physical systems (see $[4,12,17,18,23,24]$, to mention only few, and the references therein). In this paper we make an effort towards understanding the Dirac structures for infinite-dimensional systems. We consider Dirac structures on Hilbert spaces, which are general enough to cover large classes of models. Our approach uses the formalism of linear relations. In the view of the close connections with system theory, we adopt the notions of flow and effort variables. Once the space of flows and efforts are specified, it is possible to give different properties and representations of Dirac structures and investigate the relations between them.

\footnotetext{
*Corresponding author. E-mail: adrian.sandovici@luminis.ro
} 
The paper is structured as follows. The notion of Dirac structure on Hilbert spaces is recalled and some background material is presented in Section 2. A kernel representation for finite-dimensional Dirac structures (see [4]) is extended, in Section 3, in a natural way to Dirac structures on Hilbert spaces. Relations between the kernel representation and a scattering representation are given in Section 4. Finally, several illustrative examples are presented in Section 5. Further proofs are collected in the Appendix.

\section{Preliminaries}

Consider two Hilbert spaces $\mathcal{E}$ (the space of efforts) and $\mathcal{F}$ (the space of flows), and assume that there exists a unitary operator $r_{\mathcal{E}, \mathcal{F}}$ from $\mathcal{E}$ to $\mathcal{F}$. Let $\mathcal{F} \oplus \mathcal{E}$ be the Hilbert space determined by the Cartesian product space $\mathcal{F} \times \mathcal{E}$ equipped with the natural inner product:

$$
\left\langle\left(f_{1}, e_{1}\right) \mid\left(f_{2}, e_{2}\right)\right\rangle_{\mathcal{F} \oplus \mathcal{E}}=\left\langle f_{1} \mid f_{2}\right\rangle_{\mathcal{F}}+\left\langle e_{1} \mid e_{2}\right\rangle_{\mathcal{E}},
$$

where $f_{1}, f_{2} \in \mathcal{F}, e_{1}, e_{2} \in \mathcal{E}$. Further, define an indefinite inner product on $\mathcal{F} \times \mathcal{E}$, by

$$
\left[\left(f_{1}, e_{1}\right) \mid\left(f_{2}, e_{2}\right)\right]_{\mathcal{B}}=\left\langle f_{1} \mid r_{\mathcal{E}, \mathcal{F}} e_{2}\right\rangle_{\mathcal{F}}+\left\langle e_{1} \mid r_{\mathcal{F}, \mathcal{E}} f_{2}\right\rangle_{\mathcal{E}}
$$

The Cartesian product $\mathcal{F} \times \mathcal{E}$ equipped with the inner product $[\cdot \mid \cdot]_{\mathcal{B}}$ is called the bond space $\mathcal{B}$. Denote by $b=(f, e)$ an element of $\mathcal{B}$. For a linear space $\mathcal{L} \subset \mathcal{B}$ the orthogonal complement $\mathcal{L}^{[\perp]}$ of $\mathcal{L}$ is defined by

$$
\mathcal{L}^{[\perp]}=\left\{b^{\prime} \in \mathcal{B},\left[b \mid b^{\prime}\right]_{\mathcal{B}}=0, \forall b \in \mathcal{L}\right\} .
$$

Proposition 2.1. The bond space $\mathcal{B}$ is non-degenerate.

Proof. ([17, Section 2]) For any linear subspace $\mathcal{L}$ of $\mathcal{B}$ one has the equality

$$
\mathcal{L}^{[\perp]}=\left(\begin{array}{cc}
0 & r_{\mathcal{E}, \mathcal{F}} \\
r_{\mathcal{F}, \mathcal{E}} & 0
\end{array}\right)\left(\mathcal{L}^{\perp}\right)
$$

where $\mathcal{L}^{\perp}$ denotes the orthogonal complement of $\mathcal{L}$ with respect to the scalar product $(2.1)$. Therefore any orthogonal complement will be closed, and $\mathcal{B}^{[\perp]}=\{0\}$. The last equality shows that the bond space is non-degenerate.

One can now define a Dirac structure on $\mathcal{B}$.

Definition 2.2. Let $\mathcal{D}$ be a linear subspace of $\mathcal{B}$. Then $\mathcal{D}$ is said to be a Dirac structure on $\mathcal{B}$ if $\mathcal{D}=\mathcal{D}^{[\perp]}$.

In the sequel we review several known facts about linear relations on Hilbert spaces. A linear relation from a Hilbert space $\mathcal{F}$ to a Hilbert space $\mathcal{E}$ is a linear subspace $A$ of the Cartesian product $\mathcal{F} \times \mathcal{E}$. Remark that in the context of linear relations a linear operator $A$ from $\mathcal{F}$ to $\mathcal{E}$ is identified with its graph. The following self-explanatory notions domain, range, kernel, and multi-valued part of a linear relation $A$ will be used throughout the paper:

$$
\begin{aligned}
& \operatorname{dom} A=\{f \in \mathcal{F}:(f, e) \in A\}, \quad \operatorname{ran} A=\{e \in \mathcal{E}:(f, e) \in A\}, \\
& \operatorname{ker} A=\{f \in \mathcal{F}:(f, 0) \in A\}, \quad \operatorname{mul} A=\{e \in \mathcal{E}:(0, e) \in A\} .
\end{aligned}
$$

A linear relation $A$ from $\mathcal{F}$ to $\mathcal{E}$ is closed if it is closed as a subspace of the Hilbert space $\mathcal{F} \oplus \mathcal{E}$; the closure of the relation $A$ is the closure of the subspace $A$ in $\mathcal{F} \oplus \mathcal{E}$. If $A$ is closed then the subspaces $\operatorname{ker} A$ and mul $A$ are also closed. A linear relation is said to be closable if its closure is the graph of an operator. Remark that a linear relation $A$ is the graph of an operator if and only if mul $A=\{0\}$.

The adjoint of a linear relation $A$ from $\mathcal{F}$ to $\mathcal{E}$ is the closed linear relation $A^{*}$ from $\mathcal{E}$ to $\mathcal{F}$ defined by $A^{*}=\left\{\left(e^{\prime}, f^{\prime}\right) \in \mathcal{E} \times \mathcal{F}:\left\langle f^{\prime} \mid f\right\rangle_{\mathcal{F}}=\left\langle e^{\prime} \mid e\right\rangle_{\mathcal{E}}\right.$ for all $\left.(f, e) \in A\right\}$. The formal inverse $A^{-1}$ is defined as $A^{-1}=\{(e, f):(f, e) \in A\}$, and $A^{-1}$ is a linear relation from $\mathcal{E}$ to $\mathcal{F}$. One can notice that the 
following formal identities hold: $\operatorname{dom} A^{-1}=\operatorname{ran} A$, and $\operatorname{ker} A^{-1}=\operatorname{mul} A$. Notice that $\left(A^{-1}\right)^{*}=\left(A^{*}\right)^{-1}$, and so $(\operatorname{dom} A)^{\perp}=\operatorname{mul} A^{*}$, and $(\operatorname{ran} A)^{\perp}=\operatorname{ker} A^{*}$. Clearly, the double adjoint $A^{* *}$ is the closure of the relation $A$. A linear relation $A$ in a Hilbert space $\mathcal{E}$ (i.e., from $\mathcal{E}$ to $\mathcal{E}$ ) is said to be skew-symmetric if $A \subset-A^{*}$, and a linear relation $A$ in a Hilbert space $\mathcal{E}$ is said to be skew-adjoint if $A^{*}=-A$ (so that it is automatically closed).

The following example offers a class of Dirac structures.

Example 2.3. Let $\mathcal{E}$ be a Hilbert space and let $A$ be a skew-adjoint (unbounded in general) operator from $\operatorname{dom} A \subseteq \mathcal{E}$ to $\mathcal{E}$, that is

$$
\langle A x \mid y\rangle+\langle x \mid A y\rangle=0,
$$

for all $x, y \in \operatorname{dom} A=\operatorname{dom} A^{*}$. Then the graph of $A$,

$$
\mathcal{G}(A)=\{(x, A x): x \in \operatorname{dom} A\}
$$

is a Dirac structure. Indeed, the definition of a skew-adjoint operator leads to

$$
(\mathcal{G}(A))^{[\perp]}=\mathcal{G}(-A)^{*}=\mathcal{G}(A),
$$

so that the conclusion follows.

Now let $A$ and $B$ be linear relations from $\mathcal{F}$ to $\mathcal{E}$ and from $\mathcal{E}$ to $\mathcal{H}$, respectively. Then the product of $B$ and $A$ is the linear relation $B A$ from $\mathcal{F}$ to $\mathcal{H}$ defined by

$$
B A=\{(f, h) \in \mathcal{F} \times \mathcal{H}:(f, e) \in A,(e, h) \in B \text { for some } e \in \mathcal{E}\} .
$$

This definition agrees with the usual one for operators. Recall also the definition of a sum of two linear relations. For two linear relations $A_{1}$ and $A_{2}$ from $\mathcal{F}$ to $\mathcal{E}$, the operator-like sum $A_{1}+A_{2}$ is the relation from $\mathcal{F}$ to $\mathcal{E}$ defined by

$$
A_{1}+A_{2}=\left\{\left(f, e_{1}+e_{2}\right):\left(f, e_{i}\right) \in A_{i}, i=1,2\right\} .
$$

Some properties of Dirac structures are presented in the following result.

Proposition 2.4. Let $\mathcal{D}$ be a Dirac structure on $\mathcal{B}$. Then:

(i) $\operatorname{ker} \mathcal{D}=\left(\operatorname{ran}\left(r_{\mathcal{E}, \mathcal{F}} \mathcal{D}\right)\right)^{\perp}=\operatorname{ker}\left(r_{\mathcal{E}, \mathcal{F}} \mathcal{D}\right)^{*}$;

(ii) $\operatorname{mul} \mathcal{D}=\left(r_{\mathcal{F}, \mathcal{E}}(\operatorname{dom} \mathcal{D})\right)^{\perp}$.

One can state the following propositions.

Proposition 2.5. Let $\mathcal{L}$ be a linear subspace of $\mathcal{B}$. Then:

$$
\mathcal{L}^{[\perp]}=\left(\begin{array}{cc}
0 & -r_{\mathcal{E}, \mathcal{F}} \\
r_{\mathcal{F}, \mathcal{E}} & 0
\end{array}\right)\left(\mathcal{L}^{*}\right)^{-1}
$$

Proof. It follows from the definition of the adjoint relation that

$$
\left(\mathcal{L}^{*}\right)^{-1}=\left(\begin{array}{cc}
I_{\mathcal{F}} & 0 \\
0 & -I_{\mathcal{E}}
\end{array}\right)\left(\mathcal{L}^{\perp}\right)
$$

so that

$$
\mathcal{L}^{\perp}=\left(\begin{array}{cc}
I_{\mathcal{F}} & 0 \\
0 & -I_{\mathcal{E}}
\end{array}\right)\left(\mathcal{L}^{*}\right)^{-1}
$$

A combination of (2.4) and (2.7) leads to (2.5).

Using the previous proposition, one can formulate a characterization of Dirac structures in the terminology of linear relations. 
Proposition 2.6. $\mathcal{D}$ is a Dirac structure if and only if

$$
\mathcal{D}=\left(\begin{array}{cc}
0 & -r_{\mathcal{E}, \mathcal{F}} \\
r_{\mathcal{F}, \mathcal{E}} & 0
\end{array}\right)\left(\mathcal{D}^{*}\right)^{-1}
$$

Remark 2.7. In the sense of the product of linear relations the conditions in Lemma 2.6 can also be stated as follows:

$\mathcal{D}$ is a Dirac structure if and only if $\mathcal{D}=-r_{\mathcal{F}, \mathcal{E}} \mathcal{D}^{*} r_{\mathcal{F}, \mathcal{E}}$.

Proposition 2.8. Let $\mathcal{D}$ be a Dirac structure on $\mathcal{B}$. Then:

(i) $\mathcal{D}^{-1}$ is a Dirac structure on $\mathcal{B}^{-1}$;

(ii) $\mathcal{D}^{*}$ is a Dirac structure on $\mathcal{B}^{-1}$.

Proof. Assume that $\mathcal{D}$ is a Dirac structure on $\mathcal{B}$.

(i) Using the characterization of $\mathcal{D}$ in Remark 2.7 one has:

$$
\mathcal{D}^{-1}=\left(-r_{\mathcal{F}, \mathcal{E}} \mathcal{D}^{*} r_{\mathcal{F}, \mathcal{E}}\right)^{-1}=-r_{\mathcal{F}, \mathcal{E}}^{-1}\left(\mathcal{D}^{*}\right)^{-1} r_{\mathcal{F}, \mathcal{E}}^{-1}=-r_{\mathcal{E}, \mathcal{F}}\left(\mathcal{D}^{-1}\right)^{*} r_{\mathcal{E}, \mathcal{F}},
$$

which shows that $\mathcal{D}^{-1}$ is a Dirac structure on $\mathcal{B}^{-1}$.

(ii) Using again the characterization of $\mathcal{D}$ in Remark 2.7 one has $-r_{\mathcal{E}, \mathcal{F}} \mathcal{D}=\mathcal{D}^{*} r_{\mathcal{F}, \mathcal{E}}$, so that $\mathcal{D}^{*}=$ $-r_{\mathcal{E}, \mathcal{F}}\left(\mathcal{D}^{*}\right)^{*} r_{\mathcal{E}, \mathcal{F}}$, which shows that $\mathcal{D}^{*}$ is a Dirac structure on $\mathcal{B}^{-1}$.

Lemma 2.9. Let $\mathcal{D}$ be a Dirac structure on $\mathcal{B}$. Then:

$$
\begin{gathered}
\operatorname{mul} \mathcal{D}^{*}=r_{\mathcal{E}, \mathcal{F}}(\operatorname{mul} \mathcal{D}), \\
\operatorname{ker} \mathcal{D}^{*}=r_{\mathcal{F}, \mathcal{E}}(\operatorname{ker} \mathcal{D}), \\
\operatorname{ker} \mathcal{D} \perp\left(r_{\mathcal{E}, \mathcal{F}}(\operatorname{mul} \mathcal{D})\right), \\
\operatorname{mul} \mathcal{D} \perp\left(r_{\mathcal{F}, \mathcal{E}}(\operatorname{ker} \mathcal{D})\right), \\
\operatorname{ker} \mathcal{D}^{*} \perp\left(r_{\mathcal{F}, \mathcal{E}}\left(\operatorname{mul} \mathcal{D}^{*}\right)\right), \\
\operatorname{mul} \mathcal{D}^{*} \perp\left(r_{\mathcal{E}, \mathcal{F}}\left(\operatorname{ker} \mathcal{D}^{*}\right)\right) .
\end{gathered}
$$

Proof. The identities (2.8), (2.10) will be shown, the other relations can be obtained in a similar manner. In order to prove $(2.8)$ let $f \in \operatorname{mul} \mathcal{D}^{*}$, so that $(0, f) \in \mathcal{D}^{*}$. Using Remark 2.7 it follows that $(0, e) \in \mathcal{D}$ and $(e, f) \in-r_{\mathcal{E}, \mathcal{F}}$. This shows that $e=-r_{\mathcal{F}, \mathcal{E}} f \in \operatorname{mul} \mathcal{D}$, which further implies that $r_{\mathcal{F}, \mathcal{E}} f \in$ mul $\mathcal{D}$. Thus, $f \in r_{\mathcal{E}, \mathcal{F}}(\operatorname{mul} \mathcal{D})$. Consequently, mul $\mathcal{D}^{*} \subset r_{\mathcal{E}, \mathcal{F}}(\operatorname{mul} \mathcal{D})$. The converse inclusion follows similarly.

It will be shown the identity $(2.10)$. Let $f \in \operatorname{ker} \mathcal{D}$ and $f^{\prime} \in r_{\mathcal{E}, \mathcal{F}}(\operatorname{mul} \mathcal{D})$, so that $f^{\prime}=r_{\mathcal{E}, \mathcal{F}} e^{\prime}$ for some $e^{\prime} \in \operatorname{mul} \mathcal{D}$. Then $(f, 0) \in \mathcal{D}$ and $\left(0, e^{\prime}\right) \in \mathcal{D}$. It follows from $\left[(f, 0) \mid\left(0, e^{\prime}\right)\right]_{\mathcal{B}}=0$ that

$$
\left\langle f \mid r_{\mathcal{E}, \mathcal{F}} e^{\prime}\right\rangle_{\mathcal{F}}+\left\langle 0 \mid r_{\mathcal{F}, \mathcal{E}} 0\right\rangle_{\mathcal{E}}=0,
$$

which implies that $\left\langle f \mid f^{\prime}\right\rangle_{\mathcal{F}}=0$. Therefore, the relation (2.10) has been proved. The relation in (2.11) follows using similar arguments or by using the "duality" between $\mathcal{D}$ and $\mathcal{D}^{-1}$, cf. Proposition 2.8. Furthermore, the relations (2.12) and (2.13) are obtained by duality from those in (2.10) and (2.11), respectively.

Remark 2.10. In the case $\mathcal{E}=\mathcal{F}$ a Dirac structure is a skew-adjoint structure in the same Hilbert space $\mathcal{E}$.

Recall that in [13] (see also [15]) three classes of Dirac structures have been identified:

1. Completely multivalued Dirac structures which are of the form

$$
\mathcal{D}_{m u l}=\{(0, e): e \in \mathcal{E}\}
$$


2. Completely kernel Dirac structures which are of the form

$$
\mathcal{D}_{\text {ker }}=\{(f, 0): f \in \mathcal{F}\}
$$

3. Completely skew-adjoint Dirac structures which are determined by the graphs of an injective skewadjoint (not necessarily bounded) operators from $\mathcal{F}$ to $\mathcal{E}$.

These particular Dirac structures are called fundamental Dirac structures, cf. [13,15]. Under some conditions it has been shown in $[13,15]$ that a Dirac structure can be decomposed as an orthogonal sum of the previous introduced fundamental Dirac structures. However, for the purpose of this paper a weaker result is only needed.

Define the linear subspace $\mathcal{D}_{\text {mul }}=\mathcal{D} \cap(\{0\} \times \mathcal{E})$ in $\mathcal{B}$ and the linear subspace $\mathcal{E}_{\text {mul }}=\{e \in \mathcal{E}:(0, e) \in$ $\mathcal{D}\}$ in $\mathcal{E}$. Clearly, they are closed in $\mathcal{B}$ and in $\mathcal{E}$, respectively. Let $\mathcal{E}_{1}$ be the orthogonal complement of $\mathcal{E}_{m u l}$ in $\mathcal{E}$, so that $\mathcal{E}=\mathcal{E}_{m u l} \oplus \mathcal{E}_{1}$. Now define in $\mathcal{F}$ the linear subspace $\mathcal{F}_{\text {mul }}$ as

$$
\mathcal{F}_{\text {mul }}=\left\{f \in \mathcal{F}:\left\langle r_{\mathcal{E}, \mathcal{F}} e \mid f\right\rangle_{\mathcal{F}}=0, \forall e \in \mathcal{E}_{1}\right\},
$$

and then it is easy to see that $\mathcal{F}_{\text {mul }}$ is a closed subspace of $\mathcal{F}$, so that it has an orthogonal complement $\mathcal{F}_{1}$ in $\mathcal{F}$.

Lemma 2.11. Assume that $\mathcal{D}$ is a Dirac structure and let $\mathcal{D}_{\text {mul }}, \mathcal{E}_{\text {mul }}, \mathcal{F}_{\text {mul }}, \mathcal{E}_{1}$ and $\mathcal{F}_{1}$ defined as above. Assume now that

$$
r_{\mathcal{E}, \mathcal{F}}\left(\mathcal{E}_{\text {mul }}\right)=\mathcal{F}_{\text {mul }} \text {. }
$$

Then there exists a Dirac structure $\mathcal{D}_{1}$ on the bond space $\mathcal{B}_{1}:=\mathcal{F}_{1} \times \mathcal{E}_{1}$ such that

$$
\mathcal{D}=\mathcal{D}_{m u l} \oplus \mathcal{D}_{1}
$$

Proof. It follows from (2.14) that $r_{\mathcal{E}, \mathcal{F}}\left(\mathcal{E}_{1}\right)=\mathcal{F}_{1}$. Therefore $\mathcal{D}_{\text {mul }}$ is a completely multi-valued Dirac structure on the bond space $\mathcal{B}_{\text {mul }}:=\mathcal{F}_{m u l} \times \mathcal{E}_{\text {mul }}$ and there exists a Dirac structure $\mathcal{D}_{1}$ on the bond space $\mathcal{B}_{1}:=\mathcal{F}_{1} \times \mathcal{E}_{1}$ such that $\mathcal{D}=\mathcal{D}_{m u l} \oplus \mathcal{D}_{1}$. Furthermore, the Dirac structure $\mathcal{D}_{1}$ is the graph of a skew-adjoint (not necessarily bounded) operator from the Hilbert space $\mathcal{F}_{1}$ to the Hilbert space $\mathcal{E}_{1}$.

\section{A kernel representation of a Dirac structure}

Denote by $\overline{\operatorname{ran} T}$ the closure of the range of a bounded, everywhere defined linear operator $T$. Let $\mathcal{H}$ be a Hilbert space isometrically isomorphic to the Hilbert spaces $\mathcal{E}$ and $\mathcal{F}$. The definition of kernel representations for Dirac structures in the finite-dimensional case (see for instance [4]) is extended to Dirac structure on Hilbert spaces as follows.

Definition 3.1. A Dirac structure $\mathcal{D}$ on $\mathcal{B}$ is said to have a kernel representation if there exist bounded linear operators $F: \mathcal{F} \rightarrow \mathcal{H}$ and $E: \mathcal{E} \rightarrow \mathcal{H}$ such that

$$
\mathcal{D}=\{(f, e) \in \mathcal{F} \times \mathcal{E}, F f+E e=0\},
$$

and the following two conditions are satisfied

$$
\begin{gathered}
F r_{\mathcal{E}, \mathcal{F}} E^{*}+E r_{\mathcal{F}, \mathcal{E}} F^{*}=0, \\
\overline{\operatorname{ran}[F E]}=\mathcal{H} .
\end{gathered}
$$

Denote by $(F, E)_{\mathcal{D}}$ the kernel representation of $\mathcal{D}$ corresponding to $F$ and $E$. Let us consider, without restriction of generality, that $\mathcal{H}=\mathcal{F}$. 
Lemma 3.2. Assume that $\mathcal{E}$ and $\mathcal{F}$ are two Hilbert spaces and assume that there exists a unitary operator $r_{\mathcal{E}, \mathcal{F}}$ from $\mathcal{E}$ to $\mathcal{F}$.

(i) Let the linear operators $E_{\text {mul }}=O_{\mathcal{E}, \mathcal{F}}$ and $F_{\text {mul }}=I_{\mathcal{F}}$. Then the pair $\left(E_{\text {mul }}, F_{\text {mul }}\right)$ is a kernel representation for the Dirac structure $\mathcal{D}_{\text {mul }}=\{(0, e): e \in \mathcal{E}\}$.

(ii) Let the linear operators $E_{k e r}=r_{\mathcal{E}, \mathcal{F}}$ and $F_{k e r}=0_{\mathcal{F}}$. Then the pair $\left(E_{k e r}, F_{k e r}\right)$ is a kernel representation for the Dirac structure $\mathcal{D}_{\text {ker }}=\{(f, 0): f \in \mathcal{F}\}$.

Proof. It is straightforward to check that in both cases the conditions (3.1), (3.2) and (3.3) in Definition 3.1 are satisfied.

Lemma 3.3. Let $\mathcal{D}$ be a Dirac structure on $\mathcal{B}$. Define the linear relations $F$ from $\mathcal{F}$ to $\mathcal{H}$, and $E$ from $\mathcal{E}$ to $\mathcal{H}$ by

$$
\begin{gathered}
F=\left[\left(\mathcal{D}+r_{\mathcal{F}, \mathcal{E}}\right)^{-1}+\left(\mathcal{D}-r_{\mathcal{F}, \mathcal{E}}\right)^{-1}\right] r_{\mathcal{F}, \mathcal{E}}, \\
E=\left(\mathcal{D}+r_{\mathcal{F}, \mathcal{E}}\right)^{-1}-\left(\mathcal{D}-r_{\mathcal{F}, \mathcal{E}}\right)^{-1},
\end{gathered}
$$

respectively. Then $E$ and $F$ are bounded, everywhere defined linear operators.

Proof. Let $\mathcal{D}$ be a Dirac structure on $\mathcal{B}$. We show first that the linear relation $\left(\mathcal{D}+r_{\mathcal{F}, \mathcal{E}}\right)^{-1}$ is the graph of a bounded operator from $\mathcal{E}$ to $\mathcal{F}$. Let $(e, f) \in\left(\mathcal{D}+r_{\mathcal{F}, \mathcal{E}}\right)^{-1}$, so that $(f, e) \in\left(\mathcal{D}+r_{\mathcal{F}, \mathcal{E}}\right)$. Since $\left(f, r_{\mathcal{F}, \mathcal{E}} f\right) \in r_{\mathcal{F}, \mathcal{E}}$ one has $\left(f, e-r_{\mathcal{F}, \mathcal{E}} f\right) \in \mathcal{D}$. Then

$$
\left[\left(f, e-r_{\mathcal{F}, \mathcal{E}} f\right) \mid\left(f, e-r_{\mathcal{F}, \mathcal{E}} f\right)\right]_{\mathcal{B}}=0,
$$

so that $\left\langle f \mid r_{\mathcal{E}, \mathcal{F}} e\right\rangle=\|f\|^{2}$. Using Cauchy inequality one can obtain $\|f\| \leq\|e\|$, for all $(f, e) \in \mathcal{D}$. If $e=0$ then $f=0$ so that $\left(\mathcal{D}+r_{\mathcal{F}, \mathcal{E}}\right)^{-1}$ is the graph of an operator. Furthermore, the inequality $\|f\| \leq\|e\|$ for all $(f, e) \in \mathcal{D}$ shows that the operator is bounded. Using similar arguments it can be shown that $\left(\mathcal{D}-r_{\mathcal{F}, \mathcal{E}}\right)^{-1}$ is the graph of a bounded operator from $\mathcal{E}$ to $\mathcal{F}$. Consequently $E$ and $F$ are bounded, everywhere defined linear operators.

Lemma 3.4. Let $\mathcal{D}$ be a Dirac structure on $\mathcal{B}$ and assume that $\operatorname{mul} \mathcal{D}=\{0\}$. The Dirac structure $\mathcal{D}$ has the kernel representation (3.1) with the operators $F$ and $E$ given by (3.4) and (3.5), respectively.

Proof. Denote by $\mathcal{T}$ the set $\{(f, e) \in \mathcal{F} \times \mathcal{E}, F f+E e=0\}$. It will be shown that $\mathcal{D} \subset \mathcal{T}$. Let $(f, e) \in \mathcal{D}$. Since $\left(f, r_{\mathcal{F}, \mathcal{E}} f\right) \in r_{\mathcal{F}, \mathcal{E}}$ it follows that $\left(f, e+r_{\mathcal{F}, \mathcal{E}} f\right) \in \mathcal{D}+r_{\mathcal{F}, \mathcal{E}}$, therefore $\left(e+r_{\mathcal{F}, \mathcal{E}} f, f\right) \in\left(\mathcal{D}+r_{\mathcal{F}, \mathcal{E}}\right)^{-1}$. This implies that $\left(\mathcal{D}+r_{\mathcal{F}, \mathcal{E}}\right)^{-1}\left(e+r_{\mathcal{F}, \mathcal{E}} f\right)=f$, so that

$$
\left(\mathcal{D}+r_{\mathcal{F}, \mathcal{E}}\right)^{-1} e+\left(\mathcal{D}+r_{\mathcal{F}, \mathcal{E}}\right)^{-1} r_{\mathcal{F}, \mathcal{E}} f=f .
$$

Similarly one gets:

$$
\left(\mathcal{D}-r_{\mathcal{F}, \mathcal{E}}\right)^{-1} e-\left(\mathcal{D}-r_{\mathcal{F}, \mathcal{E}}\right)^{-1} r_{\mathcal{F}, \mathcal{E}} f=f .
$$

The equalities (3.6) and (3.7) lead to the $F f+E e=0$ with the operators $F$ and $E$ given by (3.4) and (3.5), respectively. Thus, $\mathcal{D} \subset \mathcal{T}$.

It will be now shown that $\mathcal{T} \subset \mathcal{D}$. Assume that $(f, e) \in \mathcal{F} \times \mathcal{E}$ such that $F f+E e=0$. Then

$$
\left(\mathcal{D}+r_{\mathcal{F}, \mathcal{E}}\right)^{-1}\left(e+r_{\mathcal{F}, \mathcal{E}} f\right)=\left(\mathcal{D}-r_{\mathcal{F}, \mathcal{E}}\right)^{-1}\left(e-r_{\mathcal{F}, \mathcal{E}} f\right)=h,
$$

for some $h \in \mathcal{F}$. Then $\left(h, e+r_{\mathcal{F}, \mathcal{E}} f\right) \in \mathcal{D}+r_{\mathcal{F}, \mathcal{E}}$ and $\left(h, e-r_{\mathcal{F}, \mathcal{E}} f\right) \in \mathcal{D}-r_{\mathcal{F}, \mathcal{E}}$, which implies that

$$
\left(h, e+r_{\mathcal{F}, \mathcal{E}} f-r_{\mathcal{F}, \mathcal{E}} h\right) \in \mathcal{D} \text {, and }\left(h, e-r_{\mathcal{F}, \mathcal{E}} f+r_{\mathcal{F}, \mathcal{E}} h\right) \in \mathcal{D} \text {. }
$$

It follows from (3.8) that

$$
\left(0,2 r_{\mathcal{F}, \mathcal{E}} f-2 r_{\mathcal{F}, \mathcal{E}} h\right)=\left(h, e+r_{\mathcal{F}, \mathcal{E}} f-r_{\mathcal{F}, \mathcal{E}} h\right)-\left(h, e-r_{\mathcal{F}, \mathcal{E}} f+r_{\mathcal{F}, \mathcal{E}} h\right) \in \mathcal{D},
$$


so that $2 r_{\mathcal{F}, \mathcal{E}}(f-h) \in \operatorname{mul} \mathcal{D}=\{0\}$. This shows that $f=h$. A combination of $(3.8)$ and $f=h$ leads to $(f, e) \in \mathcal{D}$, so that $\mathcal{T} \subset \mathcal{D}$. Thus, (3.1) has been completely proved.

It remains to show that (3.2) and (3.3) are satisfied. In order to prove (3.2) one remarks that

$$
E^{*}=\left(\mathcal{D}^{*}+r_{\mathcal{E}, \mathcal{F}}\right)^{-1}-\left(\mathcal{D}^{*}-r_{\mathcal{E}, \mathcal{F}}\right)^{-1} \text { and } F^{*}=r_{\mathcal{E}, \mathcal{F}} \cdot\left[\left(\mathcal{D}^{*}+r_{\mathcal{E}, \mathcal{F}}\right)^{-1}+\left(\mathcal{D}^{*}-r_{\mathcal{E}, \mathcal{F}}\right)^{-1}\right] .
$$

According to Remark 2.7b), the fact that $\mathcal{D}$ is a Dirac structure on $\mathcal{B}$ can be expressed by the identity $\mathcal{D}^{*}=-r_{\mathcal{E}, \mathcal{F}} \mathcal{D} r_{\mathcal{E}, \mathcal{F}}$. By a direct computation the last identity is equivalent to the identity $\left(\mathcal{D}^{*}+r_{\mathcal{E}, \mathcal{F}}\right) \cdot\left(\mathcal{D}+r_{\mathcal{F}, \mathcal{E}}\right)=\left(\mathcal{D}^{*}-r_{\mathcal{E}, \mathcal{F}}\right) \cdot\left(\mathcal{D}-r_{\mathcal{F}, \mathcal{E}}\right)$, which is also equivalent to

$$
\left(\mathcal{D}+r_{\mathcal{F}, \mathcal{E}}\right)^{-1} \cdot\left(\mathcal{D}^{*}+r_{\mathcal{E}, \mathcal{F}}\right)^{-1}=\left(\mathcal{D}-r_{\mathcal{F}, \mathcal{E}}\right)^{-1} \cdot\left(\mathcal{D}^{*}-r_{\mathcal{E}, \mathcal{F}}\right)^{-1} .
$$

Using (3.5), (3.4), the obvious identity $r_{\mathcal{E}, \mathcal{F}} \cdot r_{\mathcal{F}, \mathcal{E}}=I_{\mathcal{E}}$ and the equality (3.10), one gets (3.2) by a direct computation.

Further, it will be shown that (3.3) holds. Assume that $h \in(\operatorname{ran}[F E])^{\perp}$, so that

$$
\langle h \mid F f+E e\rangle_{\mathcal{F}}=0,
$$

for all $(f, e) \in \mathcal{F} \times \mathcal{E}$. For $e=0$ it follows from $(3.10)$ that $\langle h \mid F f\rangle_{\mathcal{F}}=0$ for all $f \in \mathcal{F}$, so that $\left\langle F^{*} h \mid f\right\rangle_{\mathcal{F}}=0$ for all $f \in \mathcal{F}$. which shows that $F^{*} h=0$. Similarly, $E^{*} h=0$. Using the formulas for $F^{*}$ and $E^{*}$ one obtains from the equalities $F^{*} h=E^{*} h=0$ that

$$
\left(\mathcal{D}^{*}+r_{\mathcal{E}, \mathcal{F}}\right)^{-1} h=\left(\mathcal{D}^{*}-r_{\mathcal{E}, \mathcal{F}}\right)^{-1} h=0
$$

It follows from $(3.11)$ that $(h, 0) \in\left(\mathcal{D}^{*}+r_{\mathcal{E}, \mathcal{F}}\right)^{-1}$, so that $(0, h) \in \mathcal{D}^{*}+r_{\mathcal{E}, \mathcal{F}}$ which shows that $(0, h) \in \mathcal{D}^{*}$. Thus, using Lemma $2.9, h \in \operatorname{mul} \mathcal{D}^{*}=r_{\mathcal{E}, \mathcal{F}}(\operatorname{mul} \mathcal{D})$, so that $h^{\prime}=r_{\mathcal{F}, \mathcal{E}} h \in \operatorname{mul} \mathcal{D}$. This further leads to $\left(0, h^{\prime}\right) \in \mathcal{D}$, so that $\left(0, h^{\prime}\right) \in \mathcal{D} \pm r_{\mathcal{F}, \mathcal{E}}$. Consequently, $\left(h^{\prime}, 0\right) \in\left(\mathcal{D} \pm r_{\mathcal{E}, \mathcal{F}}\right)^{-1}$, and then $\left(\mathcal{D} \pm r_{\mathcal{E}, \mathcal{F}}\right)^{-1} r_{\mathcal{F}, \mathcal{E}} h=0$. This leads to $F h=E\left(r_{\mathcal{F}, \mathcal{E}} h\right)=0$, so that $\left(h, r_{\mathcal{F}, \mathcal{E}} h\right) \in \mathcal{D}$. Since $\left(0, r_{\mathcal{F}, \mathcal{E}} h\right) \in \mathcal{D}$, one has $(h, 0)=\left(h, r_{\mathcal{F}, \mathcal{E}} h\right)-\left(0, r_{\mathcal{F}, \mathcal{E}} h\right) \in \mathcal{D}$. This shows that $h \in \operatorname{ker} \mathcal{D}$. Also, $h=r_{\mathcal{E}, \mathcal{F}}\left(r_{\mathcal{F}, \mathcal{E}} h\right) \in r_{\mathcal{E}, \mathcal{F}}(\operatorname{mul} \mathcal{D})$. Since $\mathcal{D}$ is a Dirac structure, one can use Lemma 2.9 to obtain $\operatorname{ker} \mathcal{D} \cap\left(r_{\mathcal{E}, \mathcal{F}}(\operatorname{mul} \mathcal{D})\right)=\{0\}$ which leads now to $h=0$. Thus, $(\operatorname{ran}[F E])^{\perp}=\{0\}$, so that (3.3) has been proved.

A particular kernel representation of a Dirac structure $\mathcal{D}$ can always be obtained directly from $\mathcal{D}$ as it is shown in the next theorem. We will refer to this representation as the "canonical kernel representation" of the Dirac structure $\mathcal{D}$.

Theorem 3.5. Let $\mathcal{D}$ be a Dirac structure on $\mathcal{B}$ and let $\mathcal{D}_{\text {mul }}, \mathcal{E}_{\text {mul }}, \mathcal{F}_{\text {mul }}, \mathcal{E}_{1}$ and $\mathcal{F}_{1}$ defined as in Section 2 Assume that

$$
r_{\mathcal{E}, \mathcal{F}}\left(\mathcal{E}_{\text {mul }}\right)=\mathcal{F}_{\text {mul }} .
$$

Then the Dirac structure $\mathcal{D}$ admits a kernel representation.

Proof. It follows from Lemma 2.11 that there exists a Dirac structure $\mathcal{D}_{1}$ on the bond space $\mathcal{B}_{1}=\mathcal{F}_{1} \times \mathcal{E}_{1}$ such that $\mathcal{D}=\mathcal{D}_{\text {mul }} \oplus \mathcal{D}_{1}$. Define the linear operators $E: \mathcal{E} \rightarrow \mathcal{F}$ and $F: \mathcal{F} \rightarrow \mathcal{F}$ by

$$
E=\left(\begin{array}{c}
E_{m u l} \\
E_{1}
\end{array}\right), \quad F=\left(\begin{array}{c}
F_{m u l} \\
F_{1}
\end{array}\right)
$$

where

$$
E_{m u l}=0_{\mathcal{E}_{m u l}, \mathcal{F}_{m u l}}, \quad F_{m u l}=I_{\mathcal{F}_{\text {mul }}}
$$

and

$$
F_{1}=\left[\left(\mathcal{D}_{1}+r_{\mathcal{F}_{1}, \mathcal{E}_{1}}\right)^{-1}+\left(\mathcal{D}_{1}-r_{\mathcal{F}_{1}, \mathcal{E}_{1}}\right)^{-1}\right] r_{\mathcal{F}_{1}, \mathcal{E}_{1}},
$$




$$
E_{1}=\left(\mathcal{D}_{1}+r_{\mathcal{F}_{1}, \mathcal{E}_{1}}\right)^{-1}-\left(\mathcal{D}_{1}-r_{\mathcal{F}_{1}, \mathcal{E}_{1}}\right)^{-1}
$$

It follows from Lemma 3.3 that $E_{1}$ and $F_{1}$ are bounded everywhere defined operators. Thus, $E$ and $F$ are bounded everywhere defined operators. Furthermore, Lemma $3.2(i)$ shows that $\left(E_{\text {mul }}, F_{m u l}\right)$ is a kernel representation for $\mathcal{D}_{\text {mul }}$ and Lemma 3.4 implies that $\left(E_{1}, F_{1}\right)$ is a kernel representation for $\mathcal{D}_{1}$. Consequently, the pair $(E, F)$ is a kernel representation for $\mathcal{D}$.

\section{Relations between the kernel representation and a scattering representation}

A scattering representation of Dirac structures for infinite dimensional spaces was basically introduced in [9] (see also [11]). Consider the Hilbert space $\mathcal{E}$ to be the scattering variable space.

For any linear subspace $\mathcal{V}$ of $\mathcal{B}$ define the linear relation $\mathcal{O}_{\mathcal{V}}$ in $\mathcal{E}$ by

$$
\mathcal{O}_{\mathcal{V}}=I_{\mathcal{E}}-2 r_{\mathcal{F}, \mathcal{E}}\left(\mathcal{V}+r_{\mathcal{F}, \mathcal{E}}\right)^{-1}
$$

Proposition 4.1. Let $\mathcal{D}$ be a Dirac structure on $\mathcal{B}$. Then $\mathcal{O}_{\mathcal{D}}$ given by (4.1) is a unitary operator in $\mathcal{E}$.

We call $\mathcal{O}_{\mathcal{D}}$ the canonical scattering operator associated to a Dirac structure $\mathcal{D}$.

Proposition 4.2. Let $\mathcal{O}$ be a unitary operator in $\mathcal{E}$. Then the linear relation

$$
\mathcal{D}_{\mathcal{O}}:=\left\{\left(\frac{1}{2} r_{\mathcal{F}, \mathcal{E}}^{-1}\left(I_{\mathcal{E}}-\mathcal{O}\right) e, \frac{1}{2}\left(I_{\mathcal{E}}+\mathcal{O}\right) e\right): e \in \mathcal{E}\right\}
$$

is a Dirac structure on $\mathcal{B}$.

Proposition 4.1 and Proposition 4.2 lead to the existence of a one-to-one correspondence between the class of Dirac structures on $\mathcal{B}$ and the class of unitary operators in $\mathcal{E}$. The above statements are known. However, using specific techniques from the theory of linear relations, alternative proofs of these two results are provided in the Appendix.

Remark 4.3. Consider $\mathcal{D}$ a Dirac structure on $\mathcal{B}$ with mul $\mathcal{D}=\{0\}$, and let $\mathcal{O}_{\mathcal{D}}$ be its canonical scattering operator. Let $(F, E)_{\mathcal{D}}$ be its corresponding canonical kernel representation. It follows from (4.1) that

$$
\left(\mathcal{D}+r_{\mathcal{F}, \mathcal{E}}\right)^{-1}=\frac{1}{2} r_{\mathcal{E}, \mathcal{F}}\left(I_{\mathcal{E}}-\mathcal{O}_{\mathcal{D}}\right)
$$

Simple computations leads to the following identity:

$$
\left(\mathcal{D}-r_{\mathcal{F}, \mathcal{E}}\right)^{-1}=\frac{1}{2} r_{\mathcal{E}, \mathcal{F}}\left(\mathcal{O}_{\mathcal{D}}{ }^{-1}-I_{\mathcal{E}}\right)
$$

Using now (3.4) and (3.5) it can be seen that:

$$
\begin{gathered}
F=\frac{1}{2} r_{\mathcal{E}, \mathcal{F}}\left(\mathcal{O}_{\mathcal{D}}{ }^{-1}-\mathcal{O}_{\mathcal{D}}\right) r_{\mathcal{F}, \mathcal{E}} . \\
E=\frac{1}{2} r_{\mathcal{E}, \mathcal{F}}\left(2 I_{\mathcal{E}}-\mathcal{O}_{\mathcal{D}}-\mathcal{O}_{\mathcal{D}}{ }^{-1}\right),
\end{gathered}
$$

with their adjoints $F^{*}=-F$ and $E^{*}=r_{\mathcal{F}, \mathcal{E}} E r_{\mathcal{F}, \mathcal{E}}$, respectively.

Conversely, given $F$ and $E$ by (3.4) and (3.5) one can write the scattering operator

$$
\mathcal{O}_{\mathcal{D}}=I_{\mathcal{E}}-r_{\mathcal{F}, \mathcal{E}} E-r_{\mathcal{F}, \mathcal{E}} F r_{\mathcal{E}, \mathcal{F}}
$$

with

$$
\mathcal{O}_{\mathcal{D}}{ }^{*}=\mathcal{O}_{\mathcal{D}}{ }^{-1}=I_{\mathcal{E}}-r_{\mathcal{F}, \mathcal{E}} E+r_{\mathcal{F}, \mathcal{E}} F r_{\mathcal{E}, \mathcal{F}}
$$


Furthermore, given a Dirac structure in a kernel representation (not necessarily the canonical one), it is possible to construct the corresponding canonical scattering operator. We need first a technical lemma.

Lemma 4.4. Let $\mathcal{D}$ be a Dirac structure on $\mathcal{B}$ with a kernel representation $(F, E)_{\mathcal{D}}$. Then

1. The operator $\operatorname{Fr}_{\mathcal{E}, \mathcal{F}}-E: \mathcal{E} \rightarrow \mathcal{H}$ is invertible.

2. The operator $r_{\mathcal{F}, \mathcal{E}} F^{*}+E^{*}: \mathcal{H} \rightarrow \mathcal{E}$ is invertible.

Proof. 1. Let $e \in \mathcal{E}$ such that $\left(F r_{\mathcal{E}, \mathcal{F}}-E\right) e=0$, which is equivalent to $F f=E r_{\mathcal{F}, \mathcal{E}} f$, where $f=r_{\mathcal{E}, \mathcal{F}} e$. This implies now that $F f+E\left(-r_{\mathcal{F}, \mathcal{E}} f\right)=0$, which leads to $\left(f,-r_{\mathcal{F}, \mathcal{E}} f\right) \in \mathcal{D}$. Consequently,

$$
\left\langle f \mid r_{\mathcal{E}, \mathcal{F}}\left(-r_{\mathcal{F}, \mathcal{E}} f\right)\right\rangle_{\mathcal{F}}+\left\langle-r_{\mathcal{F}, \mathcal{E}} f \mid r_{\mathcal{F}, \mathcal{E}} f\right\rangle_{\mathcal{E}}=0
$$

which leads to $\langle f \mid f\rangle_{\mathcal{F}}=0$. Then $f=0$, so that $e=0$.

2. From (3.2) we have that

$$
F r_{\mathcal{E}, \mathcal{F}} E^{*} h+E r_{\mathcal{F}, \mathcal{E}} F^{*} h=0 .
$$

Assume that $r_{\mathcal{F}, \mathcal{E}} F^{*} h+E^{*} h=0$ for some $h \in \mathcal{H}$. Then (4.9) leads to $\left(E E^{*}+F F^{*}\right) h=0$, which implies that $\frac{1}{2}\left\langle E E^{*} h \mid h\right\rangle_{\mathcal{H}}+\frac{1}{2}\left\langle F F^{*} h \mid h\right\rangle_{\mathcal{H}}=0$. This further shows that $E^{*} h=F^{*} h=0$. Condition (3.3) implies that $\operatorname{ker}\left[\begin{array}{l}E^{*} \\ F^{*}\end{array}\right]=\{0\}$, which gives $h=0$, and so, the operator $r_{\mathcal{F}, \mathcal{E}} F^{*}+E^{*}$ is invertible. This completes the proof.

Proposition 4.5. Let $\mathcal{D}$ be a Dirac structure on $\mathcal{B}$ with a kernel representation $(F, E)_{\mathcal{D}}$. Then the canonical scattering operator $\mathcal{O}_{\mathcal{D}}$ associated to the Dirac structure $\mathcal{D}$ is given by

$$
\mathcal{O}_{\mathcal{D}}=\left(r_{\mathcal{F}, \mathcal{E}} F^{*}-E^{*}\right)\left(r_{\mathcal{F}, \mathcal{E}} F^{*}+E^{*}\right)^{-1}=\left(F r_{\mathcal{E}, \mathcal{F}}-E\right)^{-1}\left(F r_{\mathcal{E}, \mathcal{F}}+E\right)
$$

Proof. Let $\mathcal{D}$ be a Dirac structure on $\mathcal{B}$ with a kernel representation $(F, E)_{\mathcal{D}}$. Let $\mathcal{O}_{\mathcal{D}}$ be its corresponding canonical scattering operator given by (4.1). We intend to represent $\mathcal{O}_{\mathcal{D}}$ in terms of $F$ and $E$.

According to Proposition 4.2, the Dirac structure can also be represented as

$$
\mathcal{D}_{\mathcal{O}_{\mathcal{D}}}:=\left\{\left(\frac{1}{2} r_{\mathcal{E}, \mathcal{F}}\left(I_{\mathcal{E}}-\mathcal{O}_{\mathcal{D}}\right) g, \frac{1}{2}\left(I_{\mathcal{E}}+\mathcal{O}_{\mathcal{D}}\right) g\right): g \in \mathcal{E}\right\} .
$$

Then, any $(f, e) \in \mathcal{D}$ can be written as

$$
f=\frac{1}{2} r_{\mathcal{E}, \mathcal{F}}\left(I_{\mathcal{E}}-\mathcal{O}_{\mathcal{D}}\right) g, \quad e=\frac{1}{2}\left(I_{\mathcal{E}}+\mathcal{O}_{\mathcal{D}}\right) g, g \in \mathcal{E} .
$$

Since $(F, E)_{\mathcal{D}}$ is a kernel representation of $D_{\mathcal{O}_{\mathcal{D}}}$,

$$
F f+E e=0, \forall(f, e) \in D_{\mathcal{O}_{\mathcal{D}}} .
$$

By replacing $f$ and $e$ in the last equality one obtains

$$
F r_{\mathcal{E}, \mathcal{F}}\left(I_{\mathcal{E}}-\mathcal{O}_{\mathcal{D}}\right) g+E\left(I_{\mathcal{E}}+\mathcal{O}_{\mathcal{D}}\right) g=0, \forall g \in \mathcal{E},
$$

which is equivalent to

$$
\left(F r_{\mathcal{E}, \mathcal{F}}-E\right) \mathcal{O}_{\mathcal{D}}=F r_{\mathcal{E}, \mathcal{F}}+E
$$

Now, using Lemma 4.4.1, we have

$$
\mathcal{O}_{\mathcal{D}}=\left(F r_{\mathcal{E}, \mathcal{F}}-E\right)^{-1}\left(F r_{\mathcal{E}, \mathcal{F}}+E\right) .
$$

Using $F r_{\mathcal{E}, \mathcal{F}} E^{*}+E r_{\mathcal{F}, \mathcal{E}} F^{*}=0$ (see (3.2)), one can easily write

$$
\begin{aligned}
& \left(F r_{\mathcal{E}, \mathcal{F}}+E\right)\left(r_{\mathcal{F}, \mathcal{E}} F^{*}+E^{*}\right)=F F^{*}+F r_{\mathcal{E}, \mathcal{F}} E^{*}+E r_{\mathcal{F}, \mathcal{E}} F^{*}+E E^{*} \\
& \quad=F F^{*}-F r_{\mathcal{E}, \mathcal{F}} E^{*}-E r_{\mathcal{F}, \mathcal{E}} F^{*}+E E^{*}=\left(F r_{\mathcal{E}, \mathcal{F}}-E\right)\left(r_{\mathcal{F}, \mathcal{E}} F^{*}-E^{*}\right),
\end{aligned}
$$

From the above equality, (4.12), Lemma 4.4.1 and 4.4.2 one has:

$$
\mathcal{O}_{\mathcal{D}}=\left(r_{\mathcal{F}, \mathcal{E}} F^{*}-E^{*}\right)\left(r_{\mathcal{F}, \mathcal{E}} F^{*}+E^{*}\right)^{-1} \text {. }
$$

This completes the proof. 


\section{Examples}

Example 5.1. Consider the example of the Dirac structure $\mathcal{D}$ corresponding to a infinite-dimensional circulator from [9, Example 5.2.23] defined by

$$
v_{k}+i_{k}=v_{k-1}-i_{k-1}, \quad k \in \mathbb{Z}
$$

Let

$$
\begin{gathered}
e=\left(v_{k}\right)_{k}=\left(\cdots, v_{-n}, \cdots, v_{0}, \cdots, v_{n}, \cdots\right), \\
f=\left(i_{k}\right)_{k}=\left(\cdots, i_{-n}, \cdots, i_{0}, \cdots, i_{n}, \cdots\right),
\end{gathered}
$$

$\mathcal{F}=\mathcal{E}=l^{2}(\mathbb{Z}), r_{\mathcal{E}, \mathcal{F}}=I_{l^{2}(\mathbb{Z})}$, and $[\cdot \mid \cdot]_{\mathcal{B}}$ as defined in $(2.2)$, induced by inner products on $l^{2}(\mathbb{Z})$. The operators $E$ and $F$ for the canonical kernel representation are given by (3.4) and (3.5) as follows:

$$
F=\frac{1}{2}\left(\begin{array}{cccccc}
\ddots & \ddots & \ddots & \ddots & \ddots & \ddots \\
\ddots & 0 & -1 & 0 & 0 & \ddots \\
\ddots & 1 & 0 & -1 & 0 & \ddots \\
\ddots & 0 & 1 & 0 & -1 & \ddots \\
\ddots & 0 & 0 & 1 & 0 & \ddots \\
\ddots & \ddots & \ddots & \ddots & \ddots & \ddots
\end{array}\right)
$$

and

$$
E=\frac{1}{2}\left(\begin{array}{cccccc}
\ddots & \ddots & \ddots & \ddots & \ddots & \ddots \\
\ddots & 2 & -1 & 0 & 0 & \ddots \\
\ddots & -1 & 2 & -1 & 0 & \ddots \\
\ddots & 0 & -1 & 2 & -1 & \ddots \\
\ddots & 0 & 0 & -1 & 2 & \ddots \\
\ddots & \ddots & \ddots & \ddots & \ddots & \ddots
\end{array}\right)
$$

such that

$$
\mathcal{D}=\left\{(f, e) \in l^{2}(\mathbb{Z}) \times l^{2}(\mathbb{Z}), F f+E e=0\right\} .
$$

More precisely,

$$
F\left(\left(i_{k}\right)_{k}\right)=\left(\frac{1}{2}\left(i_{k-1}-i_{k+1}\right)\right)_{k}, \text { and } E\left(\left(v_{k}\right)_{k}\right)=\left(\frac{1}{2}\left(-v_{k-1}+2 v_{k}-v_{k+1}\right)\right)_{k} .
$$

The corresponding canonical scattering operator $\mathcal{O}_{\mathcal{D}}$ is the right-shift on $l^{2}(\mathbb{Z}), \mathcal{O}_{\mathcal{D}}\left(\left(r_{k}\right)_{k}\right)=\left(\left(r_{k+1}\right)_{k}\right)$, which is an unitary operator.

Example 5.2. Consider the Hilbert spaces $\mathcal{E}=\mathcal{F}=L^{2}(0,1)$ and the operator $A$ as $A f=-f^{\prime}$ for $f \in D(A)$ where $D(A)=\left\{f \in L^{2}(0,1) \mid f^{\prime} \in L^{2}(0,1), f(0)=f(1)\right\}$ is dense in $L^{2}(0,1)$. Then

$$
\mathcal{D}=\operatorname{Graph}(A)=\left\{(f, e) \mid e=-f^{\prime}, f \in D(A)\right\}
$$

is a Dirac structure on $L^{2}(0,1) \times L^{2}(0,1)$ with $[\cdot \mid \cdot]_{\mathcal{B}}$ as defined in $(2.2)$, induced by inner products on $L^{2}(0,1)$. The operators $E$ and $F$ given by

$$
E=\left(A+I_{L^{2}(0,1)}\right)^{-1}-\left(A-I_{L^{2}(0,1)}\right)^{-1}, \quad F=\left(A+I_{L^{2}(0,1)}\right)^{-1}+\left(A-I_{L^{2}(0,1)}\right)^{-1}
$$


are well defined. For example, the equation $\left(A-i d_{L^{2}(0,1)}\right) g=u, u \in L^{2}(0,1)$, has a solution $g \in$ $D(A)$ given by $g(x)=\alpha \cdot e^{-x}-\int_{0}^{x} e^{-(x-s)} u(s) d s$, where $\alpha=\frac{\int_{0}^{1} e^{s-1} u(s) d s}{e^{-1}-1}$. Consequently, the kernel representation is

$$
\mathcal{D}=\left\{(f, e) \in\left(L^{2}(0,1)\right)^{2}, F f+E e=0, f \in D(A)\right\} .
$$

The corresponding canonical scattering operator is $\mathcal{O}_{\mathcal{D}}=\left(A-I_{L^{2}(0,1)}\right)\left(A+I_{L^{2}(0,1)}\right)^{-1}$, which is an unitary operator.

Example 5.3. Consider the Hilbert spaces $\mathcal{E}=\mathcal{F}=L^{2}(0, \infty)$, and let $r_{\mathcal{E}, \mathcal{F}}=I_{L^{2}(0, \infty)}$. Assume that $\mu>0, \mu \neq 1$. Consider also the positive function $\phi:(0, \infty) \rightarrow(0, \infty)$ given by $\phi(x)=\mu^{-\frac{1}{2}} x$ for all $x \in(0, \infty)$. Define the linear relation $\mathcal{D}$ in the Hilbert space $\mathcal{E}$ as follows:

$$
\mathcal{D}=\left\{\left(\frac{1}{2}\left(f-\mu^{-\frac{1}{4}} f \circ \phi\right), \frac{1}{2}\left(f+\mu^{-\frac{1}{4}} f \circ \phi\right)\right), f \in L^{2}(0, \infty)\right\} .
$$

It can be verified by direct computation that $\mathcal{D}$ is a Dirac structure on $\left(L^{2}(0, \infty)\right)^{2}$ with $[\cdot \mid \cdot]_{\mathcal{B}}$ as defined in $(2.2)$, induced by inner products on $L^{2}(0, \infty)$. Then the kernel operators $F, E$ from $\mathcal{E}$ to $\mathcal{E}$ are given by the following two formulas:

$$
(F f)(x)=\frac{1}{2}\left(\mu^{\frac{1}{4}} f\left(\mu^{\frac{1}{2}} x\right)-\mu^{-\frac{1}{4}} f\left(\mu^{-\frac{1}{2}} x\right)\right)
$$

and

$$
(E f)(x)=f(x)-\frac{1}{2}\left(\mu^{\frac{1}{4}} f\left(\mu^{\frac{1}{2}} x\right)+\mu^{-\frac{1}{4}} f\left(\mu^{-\frac{1}{2}} x\right)\right)
$$

for all $f \in \mathcal{E}$ and all $x \in(0, \infty)$.

The corresponding canonical scattering operator $\mathcal{O}_{\mathcal{D}}$ is the unitary scaling transformation on the Hilbert space $\mathcal{E}$ defined by

$$
\left(\mathcal{O}_{\mathcal{D}} f\right)(x)=\mu^{-\frac{1}{4}} f\left(\mu^{-\frac{1}{2}} x\right),
$$

for all $f \in \mathcal{E}$ and all $x \in(0, \infty)$. Furthermore, the bounded linear operator $\mathcal{O}_{\mathcal{D}}^{*}=\mathcal{O}_{\mathcal{D}}^{-1}$ is the unitary scaling transformation on the Hilbert space $\mathcal{E}$ defined by

$$
\left(\mathcal{O}_{\mathcal{D}}^{-1} f\right)(x)=\mu^{\frac{1}{4}} f\left(\mu^{\frac{1}{2}} x\right)
$$

for all $f \in \mathcal{E}$ and all $x \in(0, \infty)$. Since $\mathcal{O}_{\mathcal{D}}$ is a unitary operator the fact that $\mathcal{D}$ is a Dirac structure follows also from Lemma 4.2.

Example 5.4. [17, Example 3.9] Consider the Hilbert spaces $\mathcal{E}_{1}=\mathcal{F}_{1}=L^{2}(0, \infty) \oplus \mathbb{C}, \mathcal{E}_{2}=\mathcal{F}_{2}=$ $L^{2}(0, \infty)$, and consider $r_{\mathcal{E}_{1}, \mathcal{F}_{1}}=I_{L^{2}(0, \infty) \oplus \mathbb{C}}$ and $r_{\mathcal{E}_{2}, \mathcal{F}_{2}}=I_{L^{2}(0, \infty)}$. Define the linear relation $\mathcal{D}$ in the Hilbert space $\mathcal{E}=\mathcal{E}_{1} \oplus \mathcal{E}_{2}$ by

$$
\begin{aligned}
\mathcal{D}= & \left\{\left(f_{1}, f_{2}, e_{1}, e_{2}\right) \in \mathcal{E} \times \mathcal{E}: e_{1}=\left(e_{1,1}, e_{1, \partial}\right), f_{1}=\left(f_{1,1}, f_{1, \partial}\right),\right. \\
& e_{1,1} \text { and } e_{2} \text { absolutely continuous, and } \\
& \left.f_{1,1}=\frac{\partial}{\partial z} e_{2}, f_{2}=\frac{\partial}{\partial z} e_{1,1}, f_{1, \partial}=e_{2}(0), e_{1, \partial}=e_{1,1}(0)\right\} .
\end{aligned}
$$

It can be verified by direct computation that $\mathcal{D}$ is a Dirac structure on $\mathcal{E}$, with $[\cdot \mid \cdot]_{\mathcal{B}}$ as defined in $(2.2)$, induced by inner products on $\mathcal{E}$.

Define the linear operators $A$ and $B$ by

$$
A\left(\begin{array}{l}
e_{1} \\
e_{2}
\end{array}\right)=\left(\begin{array}{c}
e_{1,1}-\frac{\partial e_{2}}{\partial z} \\
e_{1,1}(0)-e_{2}(0) \\
e_{2}-\frac{\partial e_{1,1}}{\partial z}
\end{array}\right)
$$


and

$$
B\left(\begin{array}{l}
e_{1} \\
e_{2}
\end{array}\right)=\left(\begin{array}{c}
e_{1,1}+\frac{\partial e_{2}}{\partial z} \\
e_{1,1}(0)+e_{2}(0) \\
e_{2}+\frac{\partial e_{1,1}}{\partial z}
\end{array}\right)
$$

where the splitting of $e_{1}$ as given in (5.2) has been used. Then the kernel operators $F$ and $E$ from $\mathcal{E}$ to $\mathcal{E}$ are given by the following formulas:

$$
F=\frac{1}{2}\left(A B^{-1}-B A^{-1}\right)
$$

and

$$
E=I_{\mathcal{E}}-\frac{1}{2}\left(A B^{-1}+B A^{-1}\right)
$$

The corresponding canonical scattering operator $\mathcal{O}_{\mathcal{D}}$ is the unitary transformation on the Hilbert space $\mathcal{E}$ defined by

$$
\mathcal{O}_{\mathcal{D}}=B A^{-1}
$$

Appendix. Proofs of Propositions 4.1 and 4.2

Proof of Proposition 4.1. Show first that $\mathcal{O}_{\mathcal{D}}$ is an isometry in $\mathcal{E}$. Assume that $(f, e) \in \mathcal{D}$. Since $\left(f, r_{\mathcal{F}, \mathcal{E}} f\right) \in r_{\mathcal{F}, \mathcal{E}}$ it follows that $\left(f, e+r_{\mathcal{F}, \mathcal{E}} f\right) \in \mathcal{D}+r_{\mathcal{F}, \mathcal{E}}$, which implies that $\left(e+r_{\mathcal{F}, \mathcal{E}} f, f\right) \in\left(\mathcal{D}+r_{\mathcal{F}, \mathcal{E}}\right)^{-1}$. Thus,

$$
\left(e+r_{\mathcal{F}, \mathcal{E}} f,-2 r_{\mathcal{F}, \mathcal{E}} f\right) \in-2 r_{\mathcal{F}, \mathcal{E}}\left(\mathcal{D}+r_{\mathcal{F}, \mathcal{E}}\right)^{-1}
$$

Using (.1) and the obvious fact that $\left(e+r_{\mathcal{F}, \mathcal{E}} f, e+r_{\mathcal{F}, \mathcal{E}} f\right) \in I_{\mathcal{E}}$, it is obtained that

$$
\left(e+r_{\mathcal{F}, \mathcal{E}} f, e-r_{\mathcal{F}, \mathcal{E}} f\right) \in I_{\mathcal{E}}-2 r_{\mathcal{F}, \mathcal{E}}\left(\mathcal{D}+r_{\mathcal{F}, \mathcal{E}}\right)^{-1} .
$$

Furthermore, using the fact that $\mathcal{D}$ is a Dirac structure, one may write

$$
\left\|e+r_{\mathcal{F}, \mathcal{E}} f\right\|_{\mathcal{E}}^{2}-\left\|e-r_{\mathcal{F}, \mathcal{E}} f\right\|_{\mathcal{E}}^{2}=4\left\langle e \mid r_{\mathcal{F}, \mathcal{E}} f\right\rangle_{\mathcal{E}}=0
$$

which shows that $\mathcal{O}$ is an isometry, and consequently, an operator. In order to see that $\mathcal{O}$ is an unitary operator, it suffices to prove that $\operatorname{dom} \mathcal{O}=\operatorname{ran} \mathcal{O}=\mathcal{E}$. Define the operator $J: \mathcal{F} \times \mathcal{E} \rightarrow \mathcal{F} \times \mathcal{E}$ by $J(f, e)=\left(r_{\mathcal{F}, \mathcal{E}}^{-1} e, r_{\mathcal{F}, \mathcal{E}} f\right), \quad(f, e) \in \mathcal{F} \times \mathcal{E}$. Clearly, $J$ is a unitary operator in $\mathcal{F} \times \mathcal{E}$. Since $\mathcal{D}$ is a Dirac structure, it can be easily seen that $\mathcal{D}^{\perp}=J \mathcal{D}^{[\perp]}=J \mathcal{D}$, which leads to

$$
\mathcal{D} \oplus J \mathcal{D}=\mathcal{D} \oplus \mathcal{D}^{\perp}=\mathcal{F} \times \mathcal{E} .
$$

Consider any element $h \in \mathcal{E}$. Since $(0, h) \in \mathcal{F} \times \mathcal{E}$, it follows from (.3) that there exist elements $\left(f_{1}, e_{1}\right)$, $\left(f_{2}, e_{2}\right) \in \mathcal{D}$ such that $\left(f_{1}, e_{1}\right)+J\left(f_{2}, e_{2}\right)=(0, h)$. This implies that

$$
\left\{\begin{array}{l}
f_{1}+r_{\mathcal{F}, \mathcal{E}}^{-1} e_{2}=0, \\
e_{1}+r_{\mathcal{F}, \mathcal{E}} f_{2}=h,
\end{array}\right.
$$

or equivalently,

$$
\left\{\begin{array}{l}
e_{2}+r_{\mathcal{F}, \mathcal{E}} f_{1}=0 \\
e_{1}+r_{\mathcal{F}, \mathcal{E}} f_{2}=h
\end{array}\right.
$$

By summing up the equalities in (.4) one obtains that $h$ can be written in the form

$$
h=\left(e_{1}+e_{2}\right)+r_{\mathcal{F}, \mathcal{E}}\left(f_{1}+f_{2}\right),
$$

From (.2) it follows that $h \in \operatorname{dom} \mathcal{O}$. Thus, the equality $\operatorname{dom} \mathcal{O}=\mathcal{E}$ is proved. Taking the difference between the second and the first equality in (.4), it follows that $h$ can be also written in the form $h=\left(e_{1}-e_{2}\right)-r_{\mathcal{F}, \mathcal{E}}\left(f_{1}-f_{2}\right)$. Using again $(.2)$, one may conclude that $\operatorname{ran} \mathcal{O}=\mathcal{E}$. 
Proof of Proposition 4.2. Assume that $\mathcal{O}$ is a unitary operator in the Hilbert space $\mathcal{E}$. A simple computation leads to

$$
\left\langle\frac{1}{2} r_{\mathcal{F}, \mathcal{E}}^{-1}\left(I_{\mathcal{E}}-\mathcal{O}\right) e_{1} \mid \frac{1}{2} r_{\mathcal{E}, \mathcal{F}}\left(I_{\mathcal{E}}+\mathcal{O}\right) e_{2}\right\rangle_{\mathcal{F}}+\left\langle\frac{1}{2}\left(I_{\mathcal{E}}+\mathcal{O}\right) e_{1} \mid \frac{1}{2} r_{\mathcal{F}, \mathcal{E}} r_{\mathcal{F}, \mathcal{E}}^{-1}\left(I_{\mathcal{E}}-\mathcal{O}\right) e_{2}\right\rangle_{\mathcal{E}}=0
$$

for all $e_{1}, e_{2} \in \mathcal{E}$, which shows that

$$
\mathcal{D}_{\mathcal{O}} \subset \mathcal{D}_{\mathcal{O}}^{[\perp]}
$$

Consider now $(f, e) \in \mathcal{D}_{\mathcal{O}}^{[\perp]}$, so that

$$
\left[(f, e) \mid\left(\frac{1}{2} r_{\mathcal{F}, \mathcal{E}}^{-1}\left(I_{\mathcal{E}}-\mathcal{O}\right) h, \frac{1}{2}\left(I_{\mathcal{E}}+\mathcal{O}\right) h\right)\right]_{\mathcal{B}}=0
$$

for all $h \in \mathcal{E}$, or equivalently

$$
\left\langle e+r_{\mathcal{F}, \mathcal{E}} f \mid h\right\rangle_{\mathcal{E}}=\left\langle\mathcal{O}^{*}\left(e-r_{\mathcal{F}, \mathcal{E}} f\right) \mid h\right\rangle_{\mathcal{E}}=0 .
$$

Since (.6) holds for all $h \in \mathcal{E}$ it follows that $e+r_{\mathcal{F}, \mathcal{E}} f=\mathcal{O}^{*}\left(e-r_{\mathcal{F}, \mathcal{E}} f\right)$. Therefore,

$$
e-r_{\mathcal{F}, \mathcal{E}} f=\mathcal{O}\left(e+r_{\mathcal{F}, \mathcal{E}} f\right)
$$

which leads to $f=\frac{1}{2} r_{\mathcal{F}, \mathcal{E}}^{-1}\left(I_{\mathcal{E}}-\mathcal{O}\right)\left(e+r_{\mathcal{F}, \mathcal{E}} f\right)$ and $e=\frac{1}{2}\left(I_{\mathcal{E}}+\mathcal{O}\right)\left(e+r_{\mathcal{F}, \mathcal{E}} f\right)$. Consequently we have that $(f, e) \in \mathcal{D}_{\mathcal{O}}$. And so, we have proved that the following inclusion holds

$$
\mathcal{D}_{\mathcal{O}}^{[\perp]} \subset \mathcal{D}_{\mathcal{O}}
$$

A combination of $(.5)$ and $(.7)$ implies that $\mathcal{D}_{\mathcal{O}}=\mathcal{D}_{\mathcal{O}}^{[\perp]}$, which means that $\mathcal{D}_{\mathcal{O}}$ is a Dirac structure.

\section{References}

[1] R. Arens. Operational calculus of linear relations. Pacific J. Math. Vol., 11 (1961), no. 1, 9-23.

[2] T.Ya. Azizov, I.S. Iokhvidov. Linear operators in spaces with an indefinite metric. John Wiley and Sons, Chichester, 1989.

[3] J. Bognar. Indefinite inner product spaces. Springer-Verlag, Berlin-Heidelberg-New York, 1974.

[4] J. Cervera, A.J. van der Schaft, A. Baños. Interconnection of port-Hamiltonian systems and composition of Dirac structures. Automatica, 43 (2007), 212-225.

[5] T. Courant. Dirac manifolds. Tr. Am. Math. Soc., 319 (1990), 631-661.

[6] V. Derkach, S. Hassi, M. Malamud, H. de Snoo. Boundary Relations and Their Weyl Families. Tr. of Amer. Math. Soc., 358 (2006), 5351-5400.

[7] I. Dorfman. Dirac structures of integrable evolution equations. Phys. Lett. A, 125 (1987), 240-246.

[8] C. Foias, A.E. Frazho. Redheffer products and the lifting of contractions on Hilbert space. Journal of Operator Theory, 11 (1984), 193-196.

[9] G. Golo. Interconnection Structures in Port-Based Modeling: Tools for Analysis and Simulation. Ph.D. Thesis, University of Twente, September 2002.

[10] G. Golo, O.V. Iftime, A. van der Schaft. Interconnection Structures in Physical Systems: a Mathematical Formulation. In Proceedings of the $15^{\text {th }}$ International Symposium on Mathematical Theory of Networks and Systems, Notre Dame University, USA, Editors D.S. Gilliam and J. Rosenthal, 2002.

[11] G. Golo, O.V. Iftime, H. Zwart, A. van der Schaft. Tools for analysis of Dirac structures on Hilbert spaces. Memorandum no. 1729, Faculty of Applied Mathematics, University of Twente, July 2004.

[12] Y. Gorrec Le, H.J. Zwart, B. Maschke. Dirac structures and Boundary Control Systems associated with SkewSymmetric Differential Operators. SIAM J. Control and Optimization, 2005, 1864-1892.

[13] O.V. Iftime, A. Sandovici, G. Golo. Tools for analysis of Dirac Structures on Banach Spaces. In Proceedings of the 44 ${ }^{t h}$ IEEE Conference on Decision and Control and European Control Conference, Sevilla, Spain, pp. 3856-3861, December $12-15,2005$.

[14] O.V. Iftime, A. Sandovici. Interconnection of Dirac structures via kernel/image representation. In Proceedings of the American Control Conference, San Francisco, California, USA, pp. 3571-3576, June 29 - July 1, 2011.

[15] O.V. Iftime, A. Sandovici. On different types of representations of Dirac structures on Hilbert spaces. ROMAI J., 7 (2011), no. 2, 79-88. 
[16] M. Kurula, A. van der Schaft, H. Zwart. Composition of Infinite-Dimensional Linear Dirac-Type Structures. Proceedings of the 17th International Symposium on Mathematical Theory of Networks and Systems, 2006.

[17] M. Kurula, H.J. Zwart, A. van der Schaft, J. Behrndt. Dirac structure and their composition on Hilbert spaces. Journal of Mathematical Analysis and Applications, 372 (2010), 402-422.

[18] R.V. Polyuga, A. van der Schaft. Effort- and flow-constraint reduction methods for structure preserving model reduction of port-Hamiltonian systems. Systems and Control Letters, 61 (2012), 412-421.

[19] R. Redheffer. On Certain linear fractional transformations. Journal of Mathematics and Physics, 39 (1960), $269-286$.

[20] A. van der Schaft, B. Maschke. Modeling and Control of Mechanical Systems. Imperial College Press, Ch. Interconnected Mechanical Systems, part I: geometry of interconnection and implicit Hamiltonian systems, (1997), 1-15.

[21] A. van der Schaft. The Mathematics of Systems and Control: from Intelligent Control to Behavioral Systems. University of Groningen, Ch. Interconnection and Geometry, (1999), 203-217.

[22] A. van der Schaft, B. Maschke. Hamiltonian formulation of distributed parameter systems with boundary energy flow. Journal of Geometry and Physics, 42 (2002), 166-194.

[23] J. Villegas. A Port-Hamiltonian Approach to Distributed Parameter Systems. PhD Thesis, University of Twente, 2007.

[24] H. Yoshimura, H. Jacobs, J.E. Marsden. Interconnection of Dirac Structures and Lagrange-Dirac Dynamical Systems. In Proceedings of the $19^{\text {th }}$ International Symposium on Mathematical Theory of Networks and Systems, Budapest, Hungary, (2010), 5-9. 